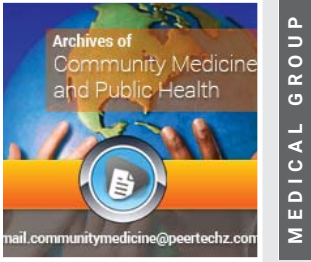

\section{Blood pressure control and}

\section{its associated factors among}

\section{hypertensive patients in federal}

teaching hospital, ido- ekiti,

\section{Southwest Nigeria}

\author{
Azeez Oyemomi lbrahim ${ }^{1 *}$, Tosin Anthony Agbesanwa ${ }^{2}$, \\ Olabode Muftau Shabi', Olusegun Emmanuel Omosanya', \\ Waheed Olalekan Ismail ${ }^{3}$ and Olabisi Olamide Deji-Dada ${ }^{2}$ \\ ${ }^{1}$ Federal Teaching Hospital, Ido Ekiti, Nigeria \\ 2Ekiti State University Teaching Hospital, Ado Ekiti, Ekiti state, Nigeria \\ ${ }^{3}$ Obafemi Awolowo University Teaching Hospital, Ile Ife, Nigeria
}

Received: 06 June, 2020

Accepted: 06 August, 2020

Published: 07 August, 2020

*Corresponding author: Azeez Oyemomi Ibrahim, MBCh B (Ife), FMC-FM, Federal Teaching Hospital, Ido Ekiti, Nigeria, Tel: +2348034139321;

E-mail: ibrahimazeez1434@gmail.com

Keywords: Blood pressure control; Associated factors Hypertensive patients; Ido- Ekiti; South West Nigeria

https://www.peertechz.com

Check for updates

\title{
Abstract
}

Background: Hypertension is a modifiable risk factor for cardiovascular disease, stroke and renal disease worldwide. Despite improvement in treatment modality and therapeutic intervention, with proven benefits in reducing the burden of uncontrolled hypertension, majority of peoples' blood pressure still remain uncontrolled.

Aim: To assess the patterns of blood pressure control and its associated factors among hypertensive patients on follow-up.

Setting: This study was done at the general out-patient department of Federal Teaching Hospital, Ido- Ekiti, Southwest Nigeria, which is a referral centre for private and government owned - health facility in the state and its environs.

Methods: Hospital-based cross sectional study was conducted from June 4 - August 31, 2019. Systematic random sampling method was used to select the 320 participants. Semi-structured interviewer- administered questionnaire was used to seek information on participants' socio-demographic characteristics, lifestyle changes and level of adherence to antihypertensive medication. Blood pressure was measured and recorded. Data was analyzed using SPSS 20 . Multivariate logistic regression analysis was used to identify the factors associated with poor blood pressure control.

Results: Mean age of the participants was $63.9 \pm 11.5$ years. $56.6 \%$ had their blood pressure controlled within the recommended limits. In multivariate analysis, civil servant $(\mathrm{OR}=45.160,95 \% \mathrm{Cl}: 6.143-332.000)$ and adherence to medication (OR=15.35, 95\% Cl: $6.303-37.391)$ were the independent associated factors with good blood pressure control while old age ( $\mathrm{OR}=0.894,95 \% \mathrm{Cl}$ : $0.837-0.955)$ and diabetes $(\mathrm{OR}=0.072,95 \% \mathrm{Cl}$ : 0.016-.0,318) were independent associated factors with poor blood pressure control.

Conclusions: Old age and diabetes are the predictors of poorly controlled blood pressure and therefore, clinicians and other stakeholders should provide close attention and targeted blood pressure control among this set of patients.

\section{Abbreviations}

FTH: Federal Teaching Hospital; AOR: Adjusted Odds Ratio; BMI: Body Mass Index; OR: Odds Ratio; CI: Confidence Interval; SPSS: Statistically Packaged for Social Sciences; BP: Blood
Pressure; SBP: Systolic Blood Pressure; DBP: Diastolic Blood Pressure; GOPD: General Outpatient Department

\section{Introduction}

Poorly controlled hypertension is a major cause of 
morbidity and mortality globally, and a modifiable risk factor for cardiovascular disease, stroke and chronic renal disease especially in Sub-Saharan Africa [1,2]. Globally, around one billion individuals are living with uncontrolled hypertension with a tendency to increase to 1.5 billion in year 2025 [3]. Uncontrolled hypertension and its complications constitute about $25 \%$ of emergency medical admissions in tertiary hospitals in Nigeria [4]. The burden of uncontrolled hypertension in developing countries has increased despite increasing awareness of its complications, due to problems of availability and accessibility to quality health care [5].

Studies conducted by Husain et al in USA revealed that the proportion of blood pressure (B.P) control was 69\% [6]. In another study by Yang et al in Seoul, Korea, proportion of blood pressure (BP) control was $84.4 \%$ [7]. Comparative prevalence of B.P control in Africa revealed 50.3\% in Ethiopia and 33.4\% in Kenya [8,9]. In Nigeria, proportion of blood pressure control ranges from $17.2-53.3 \%$ [10-13]. The current emphasis is on the control of both the systolic and diastolic blood pressure, the systolic hypertension is by far common in low socio-economic people as compared to diastolic hypertension [9]. Between 2009 and 2010 , only $50 \%$ of adult subjects with hypertension have their B.P controlled below 140/90mmHg [11].

Studies have shown that the control of B.P is still low even among the subjects who offer themselves for treatment [1114]. Reasons for poor B.P control are multi-factorials [7,1013]. Socio demographic factors, co-morbidities and poor lifestyle changes have been found to be associated with poorly controlled hypertension. [7,11,12]. Obesity, sedentary lifestyle, consumption of red meat, cigarettes smoking and alcoholic intake are the common poor lifestyle changes linked with poorly controlled hypertension [7-9].

Researchers have discovered that good management and adequate control of B.P result in reducing morbidity and mortality that are associated with cardiovascular disease and stroke $[5,6]$. Non adherence to antihypertensive medication is a significant but independent risk factor for poorly control blood pressure $[10,12]$.

Majority of recent studies on hypertension in this study area focus on the prevalence and pattern of blood pressure control, but there are paucity of data on the associated factors for poorly controlled hypertension. This study, therefore, aimed to close this knowledge gap. Findings from this study would help to create awareness on the pattern of blood pressure control and its associated factors among patients attending Federal teaching hospital (FTH), Ido- Ekiti, South West Nigeria. This will contribute to better management of this patients.

\section{Research methods and design}

\section{Study area}

FTH Ido-Ekiti is located along the federal highway linking Ekiti State to the neighbouring States of Osun, Kwara and Kogi. Ido - Ekiti is the Local Government headquatres of Ido- Osi and one of the $16^{\text {th }}$ local government areas of Ekiti State. The hospital is a referral centre for the private and government owned health facility in the state and its environs. The hospital has 180 bed facility and runs in-patients, general and specialized out- patient clinics. FTH Ido-Ekiti is an accredited centre for residency training for both West Africa College of Physician and the National Postgraduate Medical College of Nigeria.

\section{Study design and population}

The study was an hospital- based analytical cross sectional among adult hypertensive patients on follow up a General OutPatient Department (GOPD) of FTH, Ido-Ekiti.

\section{Inclusion criteria}

This includes all consenting hypertensive patients aged 18 years and above that were on medication(s) and on follow up treatment for at least 3 months.

\section{Exclusion criteria}

Hypertensive patients who were visiting the hospital for the first time and the critically ill patients that required an emergency care were excluded.

Sample size determination: This was determined using the formula [15];

$$
\mathrm{n}=\frac{\mathrm{Z}^{2}(\mathrm{P}-\mathrm{P} 1)}{\mathrm{d}^{2}} \text { and } \mathrm{nf}={ }^{\mathrm{n}} / \mathrm{N}
$$

Where $\mathrm{n}$ is the minimum sample size when the population of the participants is greater than 10,000 over a period of one year; and $\mathrm{nf}$ is the minimum sample size when the population of the participants was less than one year. $\mathrm{N}$ is the estimate of the population size of the participants in the study area over the previous one year. From the medical record of hypertensive patients attending GOPD ; N was 5,200 over a period of year 2017. $\mathrm{Z}=1.96 \% 95 \%$ Confidence Interval and $d$ is the degree of accuracy at $0.05 . \mathrm{P}$ is the estimated proportion with the attribute of interest which was $27.7 \%$ [16]. Therefore; minimum sample size $\mathrm{nf}=290$. However, $10 \%$ attrition was added for unexpected data losses and drop out during recruitment; and sample size of 320 was used for this study.

\section{Sampling technique and sampling interval}

Systematic sampling technique was used in this study. Using $\mathrm{K}=\mathrm{N} / \mathrm{n}$ where $\mathrm{k}$ was the sampling interval; $\mathrm{N}$ was the sample frame ( which was 1,600 hypertensive patients from the medical record) and $n=320$. Therefore, sampling interval $\mathrm{k}=5$.

The first participant was selected by simple random and every $5^{\text {th }}$ participant was selected by systematic random sampling until sample size of 320 participants were selected. A sticker was placed on each of the selected folders to avoid resampling at the subsequent clinics.

\section{Data collection tools and methods}

Using inclusion and exclusion criteria, eligibility of the 
participants was determined and consent was taken. The principal investigator and his team assisted by two trained resident doctors in the clinic collected the data using a pretested semi structured interviewer administered questionnaire. The pretesting of the questionnaire was conducted on 15 hypertensive patients who were selected randomly from the General Outpatient Department-(GOPD) of the Ekiti State University Teaching hospital, (EKSUTH), Ado-Ekiti and lasted for three days. The pretesting was to ascertain the fairness of the questionnaires on the participants and corrected any ambiguity. Therefore, necessary adjustment was rectified after the pretest. The questionnaire consisted of four sections: Section one assessed the socio-demographic characteristics of the participants such as age, gender, occupation, highest education attained. Other information included average daily income and duration of hypertension. Section two assessed the following lifestyle changes of the participants: alcoholic intake, cigarette smoking status, consumption of red meat, habit of salt intake and physical activity. These were all self reported. Cigarette smoking status was grouped into "never smoker", "passive smoker" or "active smoker". Alcoholic intake was either "yes" or "no." And If the answer was yes; then, follow up question was "how often?" Was it daily, weekly or monthly?. Habit of salt was either "yes" or" no" salty food. If yes; was it "added salt on cooked food" or food cooked with salt?. Physical activity was assessed by the number of minutes by day and the number of days per week a participant spent in doing physical exercise. The exercise could be in the form of trecking, street hawking ,or farming. The participant was assessed as physically active if he/she exercised more than 30 minutes per day and at least five times in a week, otherwise, as physically inactive. Section three assessed the co-morbid ailments as recorded by the Physician in the folder of the participant. Types and total number of antihypertensive medications taken by the participants were also retrieved from the folder. Section four assessed the participants' medication adherence using the Morisky's Medication Adherence Scale (MMAS-8) [17]. A score of less than 3 indicated adherence while equal or greater than 3 indicated non adherence to medication. All the questionnaires were written in English Language and translated to the local language and back translated to English language by a competent personnel in order to maintain the validity of the questionnaire.

\section{Clinical parameters of the participants}

Body Mass Index (BMI): This was determined by the weight and height of the participants using weight divided by the square of the height. The weight was measured to the nearest $0.5 \mathrm{~kg}$ using a standard weighing scale while the height was measured to the nearest $0.5 \mathrm{~cm}$ using a Standiometer. The BMI was categorized into Underweight (BMI $\left.\leq 18 \mathrm{~kg} / \mathrm{m}^{2}\right)$, Normal (BMI $18-24.9 \mathrm{~kg} / \mathrm{m}^{2}$ ), Overweight (BMI $\left.25-29.9 \mathrm{~kg} / \mathrm{m}^{2}\right)$, and Obese $\left(\mathrm{BMI} \geq 30 \mathrm{~kg} / \mathrm{m}^{2}\right)$.

Blood pressure: Blood pressure was measured using an Accosson brand of Mercury Sphygmomanometer with the appropriate cuff size. The first phase of Korotkoff sound was recorded as Systolic Blood Pressure (SBP), while the second phase, which was the disappearance of the Korotkoff sound was recorded as Diastolic Blood Pressure (DBP). The process was repeated after about 5 minutes and the average measurement of the SBP and DBP was recorded as Mean SBP and DBP for each participant. The reading of B.P was done by a Senior resident doctor. The SBP and DBP at first visit were retrieved from the folder and recorded. Blood Pressure less than or equal to $140 / 90 m m H g$ in non- diabetic and less than or equal to $130 / 80 \mathrm{mmHg}$ in diabetes was classified as controlled [18]

Data entry and analysis: Data collected were entered in SPSS Version 20.0 (Chicago, SPPS Jnc) and cross checked before analysis. Computations, tabulations of proportions, percentages and other summary statistics were done. Chisquare analysis was used to determine the significant of association. Mean value was compared with student- $t$ - test. Pearson Chi-square $\left(\mathrm{x}^{2}\right)$ and Fisher's exact test were used to compare the proportion of categorical variables. A P- value $<0.05$ was considered significant. Multiple logistic regression analysis was done for multivariate analysis with a 95\% Confidence Interval (CI) for the Odds Ratio (OR).

\section{Ethical clearance, consideration and consent}

Ethical approval was obtained from the Human Research and Ethics Committee of FTH, Ido-Ekiti before the study was conducted (ERC/2019/02/14/190A) Informed consent was obtained from all the willing participants. Confidentiality of all the information was assured by maintaining anonymity on the questionnaires. Participation was voluntary and participants were free to withdraw at anytime they so desire without losing any benefit of care or favour to those that participated. The study involved no extra cost to the participants.

\section{Results}

A total of 320 participants were studied. Their mean age was 63.9+11.5 years. (range $36-85)$. Most, 197 (61.6\%) were females and $222(70.9 \%)$ were married. One hundred and forty five $(45.0 \%)$ were traders/ artisans. Over one third, 112 $(35.0 \%)$ had tertiary education. Almost one third, 104(32.5\%) lived below the poverty level. Majority, 259 (80.9\%) have been hypertensive for more than one year with 128 (40.0\%) being over five years. There was a statistically significant association between blood pressure control and participants' marital status $(\mathrm{p}<0.001)$, occupation $(\mathrm{p}<0.001)$, education $(\mathrm{p}<0.003)$, and family history of hypertension in both parents $(p<0.006)$ (Table 1).

Only four (1.3\%) participants consumed alcohol daily and three $(0.9 \%)$ were passive cigarette smokers. Nearly half, $148(46.3 \%)$ consumed red meat regularly. Most, 211 $(65.9 \%)$ cooked their food with salt. Majority, 240 (75.0\%) were classified as inactive with $116(36.2 \%)$ being sedentary. Participants with overweight and obesity were 108 (33.7\%) and $53(16.6 \%)$ respectively. There was a statistically significant association between blood pressure control and smoking ( $\mathrm{p}<$ $0.047)$, salty food $(\mathrm{p}<0.001)$ and BMI $(\mathrm{P}<0.04)$ (Table 2$)$. Diabetes mellitus 111 (34.7\%) and Osteoarthritis 104 (32.5\%) were the common co-morbid ailments. Other co-morbid ailments were dyslipidaemia, 50 (15.6\%), PUD $14(15.0 \%)$ and Obesity $47(14.7 \%)$. There was a statistically significant 
association between blood pressure control and diabetes ( $\mathrm{p}<$ 0.001 ), obesity $(\mathrm{p}<0.001)$ and PUD ( $\mathrm{P}<0.031)$, (Table 3$)$

Regularly prescribed antihypertensive medication was calcium channel blocker (CCB), $284(88.8 \%)$ followed by Diuretics, $216(67.5 \%)$ and ACEI, 183 (57.2\%), Table 4 Most, $239(74.7 \%)$ of the participants were adherent to their medication(s). There was a statistically significant association between B.P control and medication adherence $(\mathrm{P}<0.001)$, Table 5.

In all the 320 participants, $111(34.7 \%)$ were diabetic hypertensive and 209 (65.3\%) were non diabetic hypertensive. Among the diabetic, $47(42.3 \%)$ had their B.P control at less

Table 1: Association between socio-demographic characteristics and BP control (WHO criteria).

\begin{tabular}{|c|c|c|c|c|c|}
\hline \multirow{2}{*}{ Variables } & \multicolumn{2}{|c|}{ BP control $n(\%)$} & \multirow{2}{*}{ Total } & \multirow{2}{*}{$x^{2 * *}$} & \multirow{2}{*}{$P$ value } \\
\hline & Controlled & Uncontrolled & & & \\
\hline \multicolumn{6}{|l|}{ Age group (yrs) } \\
\hline$<40$ & $8(100.0)$ & $0(0.0)$ & $8(100.0)$ & 22.069 & $<0.001$ \\
\hline $40-49$ & $28(87.5)$ & $4(12.5)$ & $32(100.0)$ & & \\
\hline $50-59$ & $30(47.6)$ & $33(52.4)$ & $63(100.0)$ & & \\
\hline $60-69$ & 59 (51.3) & $56(48.7)$ & $115(100.0)$ & & \\
\hline$\geq 70$ & $56(54.9)$ & $46(45.1)$ & $102(100.0)$ & & \\
\hline Mean \pm SD & $61.2 \pm 12.6$ & $66.0 \pm 9.5$ & $63.9 \pm 11.5$ & -3.349 & $0.001 *$ \\
\hline \multicolumn{6}{|l|}{ Sex } \\
\hline Male & $76(61.8)$ & $47(38.2)$ & $123(100.0)$ & 2.221 & 0.136 \\
\hline Female & $105(53.3)$ & $92(46.7)$ & $197(100.0)$ & & \\
\hline \multicolumn{6}{|l|}{ Marital status } \\
\hline Single & $2(100.0)$ & $0(0.0)$ & $2(100.0)$ & 11.144 & 0.011 \\
\hline Married & $140(61.7)$ & $87(38.3)$ & $227(100.0)$ & & \\
\hline Separated & $2(33.3)$ & $4(66.7)$ & $6(100.0)$ & & \\
\hline Widow & 37 (43.5) & $48(56.5)$ & $85(100.0)$ & & \\
\hline \multicolumn{6}{|l|}{ Occupation } \\
\hline Civil servants & $67(77.0)$ & $20(23.0)$ & $87(100.0)$ & 25.756 & $<0.001$ \\
\hline Traders / artisans & $62(43.1)$ & $82(56.9)$ & $144(100.0)$ & & \\
\hline Farmers & $18(60.0)$ & $12(40.0)$ & $30(100.0)$ & & \\
\hline Retirees & $23(59.0)$ & $16(41.0)$ & $39(100.0)$ & & \\
\hline Unemployed & $11(55.0)$ & $9(45.0)$ & $20(100.0)$ & & \\
\hline \multicolumn{6}{|l|}{ Education } \\
\hline None & 39 (52.7) & $35(47.3)$ & $74(100.0)$ & 16.007 & 0.003 \\
\hline Primary & $44(51.8)$ & $41(48.2)$ & $85(100.0)$ & & \\
\hline Secondary & $12(34.3)$ & $23(65.7)$ & $35(100.0)$ & & \\
\hline Tertiary & $75(67.0)$ & $37(33.0)$ & $112(100.0)$ & & \\
\hline Postgraduate & $11(78.6)$ & $3(21.4)$ & $14(100.0)$ & & \\
\hline \multicolumn{6}{|l|}{ Religion } \\
\hline Christianity & $166(56.5)$ & $128(43.5)$ & $294(100.0)$ & 0.015 & 0.903 \\
\hline Islam & $15(57.7)$ & $11(42.3)$ & $26(100.0)$ & & \\
\hline \multicolumn{6}{|l|}{ Ethnicity } \\
\hline Yoruba & $177(56.7)$ & $135(43.3)$ & $312(100.0)$ & 0.144 & 0.705 \\
\hline Ibo & $4(50.0)$ & $4(50.0)$ & $8(100.0)$ & & \\
\hline \multicolumn{6}{|l|}{ Domicile } \\
\hline Rural & $83(53.5)$ & $72(46.5)$ & $155(100.0)$ & 1.112 & 0.292 \\
\hline Urban & $98(59.4)$ & $67(40.6)$ & $165(100.0)$ & & \\
\hline \multicolumn{6}{|l|}{ Income } \\
\hline < NGN 500 / day & $53(51.0)$ & $51(49.0)$ & $104(100.0)$ & 1.967 & 0.161 \\
\hline$\geq$ NGN 500 / day & $128(59.3)$ & $88(40.7)$ & $216(100.0)$ & & \\
\hline \multicolumn{6}{|c|}{ Duration of hypertension } \\
\hline$<6$ months & $19(76.0)$ & $6(24.0)$ & $25(100.0)$ & 4.575 & 0.206 \\
\hline 6 - 12 months & $18(50.0)$ & $18(50.0)$ & $36(100.0)$ & & \\
\hline 1 - 5 years & $73(55.7)$ & $58(44.3)$ & $131(100.0)$ & & \\
\hline$>5$ years & $71(55.5)$ & $57(44.5)$ & $128(100.0)$ & & \\
\hline
\end{tabular}

Table 2: Association between BP control and lifestyle changes.

\begin{tabular}{|c|c|c|c|c|c|}
\hline \multirow{2}{*}{ Variables } & \multicolumn{2}{|c|}{ BP control n (\%) } & \multirow{2}{*}{ Total } & \multirow{2}{*}{$x^{2 * *}$} & \multirow{2}{*}{$P$ value } \\
\hline & Controlled & Uncontrolled & & & \\
\hline \multicolumn{6}{|c|}{ Frequency of alcohol intake } \\
\hline None & $154(56.4)$ & $119(43.6)$ & $273(100.0)$ & 6.149 & 0.188 \\
\hline Once a month & $11(68.8)$ & $5(31.3)$ & $16(100.0)$ & & \\
\hline Once weekly & $4(36.4)$ & $7(63.6)$ & $11(100.0)$ & & \\
\hline Thrice a week & $8(50.0)$ & $8(50.0)$ & $16(100.0)$ & & \\
\hline Everyday & $4(100.0)$ & $0(0.0)$ & $4(100.0)$ & & \\
\hline \multicolumn{6}{|l|}{ Smoking } \\
\hline No & $181(57.1)$ & $136(42.9)$ & $317(100.0)$ & 3.943 & 0.047 \\
\hline Yes (passive) & $0(0.0)$ & $3(100.0)$ & $3(100.0)$ & & \\
\hline \multicolumn{6}{|l|}{ Red meat } \\
\hline None & $21(63.6)$ & $12(36.4)$ & $33(100.0)$ & 0.879 & 0.644 \\
\hline Occasional & $79(56.8)$ & $60(43.2)$ & $139(100.0)$ & & \\
\hline Regularly & $81(54.7)$ & $67(45.3)$ & $148(100.0)$ & & \\
\hline \multicolumn{6}{|l|}{ Salty food } \\
\hline None & $12(33.3)$ & $24(66.7)$ & $36(100.0)$ & 25.167 & $<0.001$ \\
\hline Food cooked with salt & $140(66.4)$ & $71(33.6)$ & $211(100.0)$ & & \\
\hline $\begin{array}{l}\text { Add salt on cooked } \\
\text { food }\end{array}$ & $9(47.4)$ & $10(52.6)$ & 19 (100.0) & & \\
\hline $\begin{array}{l}\text { Both (Cooked and } \\
\text { Added) }\end{array}$ & $20(37.0)$ & $34(63.0)$ & $54(100.0)$ & & \\
\hline \multicolumn{6}{|l|}{ Level of activity } \\
\hline $\begin{array}{c}>30 \mathrm{~min} / \text { day, }>3 \text { times } \\
\text { / week }\end{array}$ & $52(65.0)$ & $28(35.0)$ & $80(100.0)$ & 3.853 & 0.146 \\
\hline $\begin{array}{c}<30 \mathrm{~min} / \text { day },<3 \text { times } \\
\text { / week }\end{array}$ & 70 (56.5) & $54(43.5)$ & $124(100.0)$ & & \\
\hline None & $59(50.9)$ & $57(49.1)$ & $116(100.0)$ & & \\
\hline \multicolumn{6}{|l|}{ BMI } \\
\hline Underweight & $2(40.0)$ & $3(60.0)$ & $5(100.0)$ & 8.291 & 0.040 \\
\hline Normal & $92(59.7)$ & $62(40.3)$ & $154(100.0)$ & & \\
\hline Overweight & $66(61.1)$ & $42(38.9)$ & $108(100.0)$ & & \\
\hline Obesity & $21(39.6)$ & $32(60.4)$ & $53(100.0)$ & & \\
\hline $\mathrm{BMI}(\mathrm{Kg} / \mathrm{m} 2)$ & $\begin{array}{c}25.56 \pm \\
3.91\end{array}$ & $26.78 \pm 5.82$ & $\begin{array}{c}26.09 \pm \\
4.87\end{array}$ & -2.129 & 0.034 * \\
\hline Weight (kg) & $65.9 \pm 10.2$ & $68.3 \pm 15.2$ & $66.9 \pm 12.7$ & -1.592 & $0.113^{*}$ \\
\hline Height (m) & $1.6 \pm 0.0$ & $1.6 \pm 0.1$ & $1.6 \pm 0.1$ & 1.594 & $0.112^{*}$ \\
\hline
\end{tabular}

or equal to $130 / 80 \mathrm{mmHg}$, while $64(57.7 \%)$ were uncontrolled. Almost half, $49(44.1 \%)$ had their SBP controlled while 73 (65.8\%) had their DBP controlled as well. Also, among the non diabetic- hypertensive, 134 (64.1\%) had their B.P controlled while $75(35.9 \%)$ uncontrolled. Majority 145 (69.4\%) had their SBP controlled while most, $156(74.6 \%)$ had their DBP controlled as well. (Table 6).

In the combined analysis for both the diabetic and nondiabetic hypertensive participants, the proportion of good B.P control was, 181 (56.6\%) and uncontrolled was 139 (43.4), (Figure 1). Using multivariate logistic regression analysis for B.P control, Advanced age (OR, 0.894; 95\% CI, (0.837-0.955) and diabetes (OR, 0.072; 95\% CI, (0.016-0.318) were factors associated with poor B.P control (Table 7).

\section{Discussion}

In this study, $56.6 \%$ of the hypertensive patients on follow up at FTH, Ido - Ekiti, had their blood pressure controlled within the recommended limits. However, this level of blood pressure control was better than $\mathbf{1 7 . 2 \%}$ in Enugu, South East Nigeria [10], 24.2\% in Port Harcourt, South South Nigeria[14],

Citation: Ibrahim AO, Agbesanwa TA, Shabi OM, Omosanya OE, Ismail WO, et al. (2020) Blood pressure control and its associated factors among hypertensive patients in federal teaching hospital, ido- ekiti, Southwest Nigeria. Arch Community Med Public Health 6(2): 167-173. DOI: https://dx.doi.org/10.17352/2455-5479.000099 
34.5\% in Kano, North West Nigeria [12], 46.4\% in Abeokuta, South West Nigeria[11] and 53.3\% in Ilorin, North Central Nigeria[13]. Our finding was also better than the reported findings in other African countries with $33.4 \%$ in Kenya [9] and $50.3 \%$ in Ethiopia [8]. The relatively high proportion in this study might be due to better adherence among the studied participants. Poor adherence to therapeutic plans has been identified as factor contributing to poor B.P control $[11,13]$. Adherence to antihypertensive medication in this study was $74.7 \%$ as compared to $53.0 \%$ found in Kano [12] and $60.5 \%$ in Ethiopia [8]. Antihypertensive medications decrease and regulate high blood pressure by increasing vasodilatation and decreasing vasoconstriction with resultant increase in urinary output [18]. Proportion of blood pressure control in this study was lower than $69.0 \%$ reported in USA [6] and $84.4 \%$ in Korea [7]. The differences with our finding could be due to many hypertensive patients still pay out of pocket in a low resource setting as found in the area of study.

In this study, multivariate analysis revealed that civil servants and adherence to medication are associated with good B.P control. Being a civil servant provides a source of income

Table 3: Association between BP control and Co-morbid ailments.

\begin{tabular}{|c|c|c|c|c|c|}
\hline \multirow{2}{*}{ Variables } & \multicolumn{2}{|c|}{ BP control $n(\%)$} & \multirow{2}{*}{ Total } & \multirow{2}{*}{$x^{2 *}$} & \multirow{2}{*}{$P$ value } \\
\hline & Controlled & Uncontrolled & & & \\
\hline \multicolumn{6}{|l|}{ Diabetes } \\
\hline Yes & $47(42.3)$ & $64(57.7)$ & $111(100.0)$ & 13.988 & $<0.001$ \\
\hline No & $134(64.1)$ & $75(35.9)$ & $209(100.0)$ & & \\
\hline \multicolumn{6}{|c|}{ Chronic renal disease } \\
\hline Yes & $0(0.0)$ & $2(100.0)$ & $2(100.0)$ & 2.621 & 0.105 \\
\hline No & $181(56.9)$ & $137(43.1)$ & $318(100.0)$ & & \\
\hline \multicolumn{6}{|c|}{ Dyslipidaemia } \\
\hline Yes & $22(44.0)$ & $28(56.0)$ & $50(100.0)$ & 3.806 & 0.051 \\
\hline No & $159(58.9)$ & $111(41.1)$ & $270(100.0)$ & & \\
\hline \multicolumn{6}{|l|}{ Obesity } \\
\hline Yes & $14(29.8)$ & $33(70.2)$ & $47(100.0)$ & 16.075 & $<0.001$ \\
\hline No & $167(61.2)$ & $106(38.8)$ & $273(100.0)$ & & \\
\hline \multicolumn{6}{|c|}{ Osteoarthritis } \\
\hline Yes & $52(50.0)$ & $52(50.0)$ & $104(100.0)$ & 2.701 & 0.100 \\
\hline No & $129(59.7)$ & $87(40.3)$ & $216(100.0)$ & & \\
\hline \multicolumn{6}{|l|}{ PUD } \\
\hline Yes & $34(70.8)$ & $14(29.2)$ & $48(100.0)$ & 4.681 & 0.031 \\
\hline No & $147(54.0)$ & $125(46.0)$ & $272(100.0)$ & & \\
\hline \multicolumn{6}{|l|}{ Asthma } \\
\hline Yes & $4(57.1)$ & $3(42.9)$ & $7(100.0)$ & 0.001 & 0.975 \\
\hline No & $177(56.5)$ & $136(43.5)$ & $313(100.0)$ & & \\
\hline \multicolumn{6}{|c|}{ Other comorbidity } \\
\hline Yes & $29(65.9)$ & $15(34.1)$ & $44(100.0)$ & 1.814 & 0.178 \\
\hline No & $152(55.1)$ & $124(44.9)$ & $276(100.0)$ & & \\
\hline
\end{tabular}

*Pearson chi square

Table 4: Anti-hypertensive medications.

\begin{tabular}{|c|c|c|}
\hline Variables & Frequency & Percent \\
\hline CCB & 284 & 88.8 \\
\hline Diuretics & 216 & 67.5 \\
\hline ACEI & 183 & 57.2 \\
\hline Methyldopa & 20 & 6.3 \\
\hline ARD & 17 & 5.3 \\
\hline Beta blockers & 15 & 4.7 \\
\hline
\end{tabular}

Table 5: Association between BP control and Adherence to Anti-hypertensive medications.

\begin{tabular}{|c|c|c|c|c|c|}
\hline \multirow{2}{*}{$\begin{array}{c}\text { Grades of Adherence } \\
\text { Medication Non- } \\
\text { Adherence }\end{array}$} & \multicolumn{2}{|c|}{ BP control $\boldsymbol{n}(\%)$} & \multirow{2}{*}{ Total } & \multirow{2}{*}{$\mathbf{X}^{*}$} & \multirow{2}{*}{ P value } \\
\cline { 2 - 4 } & & & & & \\
\hline Adherent & $165(69.0)$ & $74(31.0)$ & $239(100.0)$ & 59.808 & $<0.001$ \\
\hline Non-Adherent & $16(19.8)$ & $65(80.2)$ & $81(100.0)$ & & \\
\hline Total & $181(56.6)$ & $139(43.4)$ & $320(100.0)$ & & \\
\hline *Pearson chi square & & & & & \\
\hline
\end{tabular}

Table 6: Patterns of BP control in diabetics $(\leq 130 / 80 \mathrm{mmHg})$ and Non- Diabetics ( $\leq$ $140 / 90 \mathrm{mmHg}$ ).

\begin{tabular}{|c|c|c|c|c|c|}
\hline \multirow[b]{2}{*}{ Variables } & \multicolumn{2}{|c|}{$\operatorname{Sex} n(\%)$} & \multirow{2}{*}{$\begin{array}{c}\text { Total } \\
(\mathbf{N}= \\
111)\end{array}$} & \multirow[b]{2}{*}{$x^{2}$} & \multirow[b]{2}{*}{$P$ value } \\
\hline & $\begin{array}{l}\text { Male } \\
(n=39)\end{array}$ & $\begin{array}{l}\text { Female } \\
(n=72)\end{array}$ & & & \\
\hline
\end{tabular}

BP control $(\leq 130 / 80 \mathrm{mmHg})$

\begin{tabular}{|c|c|c|c|c|c|}
\hline Controlled & $\begin{array}{c}22 \\
(56.4)\end{array}$ & $25(34.7)$ & $\begin{array}{c}47 \\
(42.3)\end{array}$ & 4.874 & 0.027 \\
\hline Uncontrolled & $\begin{array}{c}17 \\
(43.6)\end{array}$ & $47(65.3)$ & $\begin{array}{c}64 \\
(57.7)\end{array}$ & & \\
\hline \multicolumn{6}{|c|}{ SBP control $(\leq 130 \mathrm{mmHg})$} \\
\hline Controlled & $\begin{array}{c}24 \\
(61.5)\end{array}$ & $25(34.7)$ & $\begin{array}{c}49 \\
(44.1)\end{array}$ & 7.378 & 0.007 \\
\hline Uncontrolled & $\begin{array}{c}15 \\
(38.5)\end{array}$ & $47(65.3)$ & $\begin{array}{c}62 \\
(55.9)\end{array}$ & & \\
\hline \multicolumn{6}{|c|}{ DBP control $(\leq 80 \mathrm{mmHg})$} \\
\hline Controlled & $\begin{array}{c}25 \\
(64.1)\end{array}$ & $48(66.7)$ & $\begin{array}{c}73 \\
(65.8)\end{array}$ & 0.074 & 0.786 \\
\hline Uncontrolled & $\begin{array}{c}14 \\
(35.9)\end{array}$ & $24(33.3)$ & $\begin{array}{c}38 \\
(34.2)\end{array}$ & & \\
\hline \multicolumn{6}{|c|}{ ontrol $(<140 / 90 \mathrm{mmHg})$} \\
\hline Controlled & $\begin{array}{c}54 \\
(64.3)\end{array}$ & $80(64.0)$ & $\begin{array}{c}134 \\
(64.1)\end{array}$ & 0.002 & 0.966 \\
\hline Uncontrolled & $\begin{array}{c}30 \\
(35.7)\end{array}$ & $45(36.0)$ & $\begin{array}{c}75 \\
(35.9)\end{array}$ & & \\
\hline \multicolumn{6}{|c|}{ control $(<140 \mathrm{mmHg})$} \\
\hline Controlled & $\begin{array}{c}62 \\
(73.8)\end{array}$ & $83(66.4)$ & $\begin{array}{c}145 \\
(69.4)\end{array}$ & 1.298 & 0.255 \\
\hline Uncontrolled & $\begin{array}{c}22 \\
(26.2)\end{array}$ & $42(33.6)$ & $\begin{array}{c}64 \\
(30.6)\end{array}$ & & \\
\hline
\end{tabular}

DBP control (<90 $\mathrm{mmHg})$

\begin{tabular}{|l|c|c|c|c|c|}
\hline Controlled & $\begin{array}{c}66 \\
(78.6)\end{array}$ & $90(72.0)$ & $\begin{array}{c}156 \\
(74.6)\end{array}$ & 1.146 & 0.284 \\
\hline Uncontrolled & $\begin{array}{c}18 \\
(21.4)\end{array}$ & $35(28.0)$ & $\begin{array}{c}53 \\
(25.4)\end{array}$ & & \\
\hline
\end{tabular}

*Pearson chi square

\section{Patern of Blood Pressure}

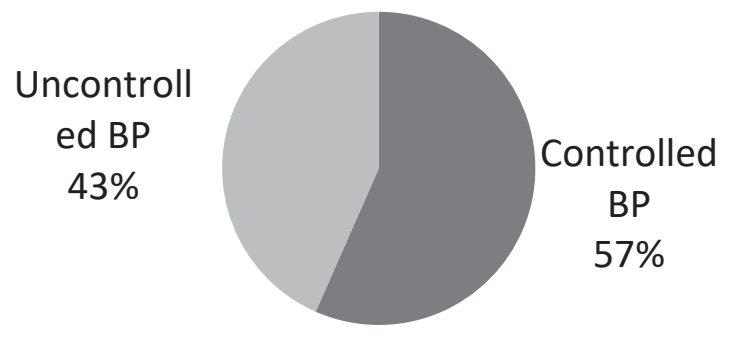

Figure 1: Patern of Blood Pressure.

Citation: Ibrahim AO, Agbesanwa TA, Shabi OM, Omosanya OE, Ismail WO, et al. (2020) Blood pressure control and its associated factors among hypertensive patients in federal teaching hospital, ido- ekiti, Southwest Nigeria. Arch Community Med Public Health 6(2): 167-173. DOI: https://dx.doi.org/10.17352/2455-5479.000099 
Table 7: Logistic regression analysis for predictors of BP control.

\begin{tabular}{|c|c|c|c|c|}
\hline Variables & B & S.E. & OR $(95 \% \mathrm{Cl})$ & $P$ value \\
\hline Age $^{*}$ & -0.112 & 0.033 & $0.894(0.837-0.955)$ & 0.001 \\
\hline \multicolumn{5}{|l|}{ Occupation } \\
\hline Civil servants & 3.810 & 1.018 & $45.160(6.143-332.000)$ & 0.000 \\
\hline Traders / artisans & 1.290 & 0.709 & $3.633(0.905-14.585)$ & 0.069 \\
\hline Farmers & 0.404 & 0.722 & $1.498(0.364-6.162)$ & 0.576 \\
\hline Retirees & 3.473 & 1.017 & $32.247(4.397-236.511)$ & 0.001 \\
\hline Unemployed & & & 1 & \\
\hline \multicolumn{5}{|l|}{ Education } \\
\hline None & 0.267 & 0.980 & $1.306(0.191-8.918)$ & 0.785 \\
\hline Primary & 0.302 & 0.912 & $1.352(0.226-8.075)$ & 0.741 \\
\hline Secondary & -2.321 & 0.981 & $0.098(0.014-0.672)$ & 0.118 \\
\hline Tertiary & -2.483 & 0.962 & $0.084(0.013-0.550)$ & 0.110 \\
\hline Postgraduate & & & 1 & \\
\hline \multicolumn{5}{|l|}{ Salty food } \\
\hline None & -2.100 & 0.729 & $0.122(0.029-0.511)$ & 0.104 \\
\hline Food cooked with salt & 0.317 & 0.515 & $1.373(0.501-3.768)$ & 0.538 \\
\hline Add salt on cooked food & -1.001 & 0.949 & $0.367(0.057-2.359)$ & 0.291 \\
\hline Both (Cooked and Added) & & & 1 & \\
\hline \multicolumn{5}{|l|}{ Diabetes } \\
\hline Yes & -2.630 & 0.758 & $0.072(0.016-0.318)$ & 0.001 \\
\hline No & & & 1 & \\
\hline \multicolumn{5}{|l|}{ PUD } \\
\hline Yes & 1.281 & 1.352 & $3.600(0.254-50.948)$ & 0.343 \\
\hline No & & & 1 & \\
\hline \multicolumn{5}{|l|}{ CCB } \\
\hline Yes & -0.688 & 1.216 & $0.502(0.046-5.442)$ & 0.571 \\
\hline No & & & 1 & \\
\hline \multicolumn{5}{|l|}{ ARD } \\
\hline Yes & 1.641 & 1.697 & $5.160(0.185-143.685)$ & 0.334 \\
\hline No & & & 1 & \\
\hline \multicolumn{5}{|l|}{ Medication Non-Adherence } \\
\hline Adherent & 2.731 & 0.454 & $15.351(6.303-37.391)$ & 0.000 \\
\hline Non-Adherent & & & 1 & \\
\hline
\end{tabular}

to procure medication and also to come to the hospital for follow up. Study has established that majority of civil servant subjects are likely to be enlightened about the need to have proper health seeking behaviour irrespective of their gender [19]. Retirees subjects are linked to good B.P control because they are likely to have more time to take care of their health due to less demands of daily work.

Adherence to anti-hypertension medication has been linked to good B.P control in this study and is related to findings from previous studies $[11,13]$. The terms adherence is comprehensive and reflect both taking the medication as prescribed (compliance) and continuing to take the medication for the duration required (persistence) [13]. Therefore, clinicians should use the opportunity of every encounter with hypertensive subjects to provide effective health education and screening for adherence as a way to reduce cardiovascular risk associated with uncontrolled hypertension.

In this study, multivariate analysis revealed that advanced age and diabetes were significant contributors to poor B.P control. Old age has been identified as an important risk factor for poor B.P control in a number of studies [8,9,12]. Elderly populations were more likely to have multiple co-morbidities as shown in this study and therefore may significantly affect their level of blood pressure control [8]. Previous studies have linked advanced age to a decrease in systemic arterial elasticity that culminated in an increase in systolic blood pressure $[8,12]$. This finding may suggest that Physicians who see after elderly hypertensive patients should also seek for and address other medical challenges that may be hiding in the patient.

Diabetic co-morbidity had a significant association with poorly controlled B.P in this study. This is consistent with previous studies. [11,13]. The prevalence of diabetes among the various identified co-morbid ailments in this study was $34.7 \%$. This may be due to greater proportion of overweight and obesity as well as age of the study participants. In this study, the control of B.P among the diabetic hypertensive participants was $42.3 \%$. This finding was poor considering the fact that adequate B.P control is of particular concern in patient with diabetes, as hypertension increases morbidity and mortality associated with stroke and cardiovascular diseases [11]. However, finding from this study was better than the reported findings of $18.4 \%$ in Abeokuta and $23.7 \%$ in Ilorin $[11,13]$. The differences may be due to high burden of co-morbidities and non adherence to antihypertensive medication. Previous study has shown that diabetic hypertensive patients required multiple drugs and this may result in additional burden, both in terms of medication adherence and high cost of the drug especially in low resource setting [11]. Other study has stated that poor B.P control in diabetic patient might be due to microvascular and macrovascular complications [20]. In this study, only $44.1 \%$ of the diabetics hypertensive participants had their SBP controlled, indicating a higher long term risk of cardiovascular disease [11,20]. This finding is important given the existing evidence that SBP is more important than DBP in predicting cardiovascular and renal disease in an ageing population $[9,11]$. The relevance of good B.P control have also been documented by several studies $[8,9,11]$. The outcome of this finding confirmed that achieving optimal B.P control in diabetic hypertensive patients is often challenging and difficulty; as only a few patients with diabetes had their B.P control below the recommended goal. This finding reinforces the need for close attention and adequate B.P control in diabetic patients.

\section{Conclusions}

The proportion of hypertensive patients with optimal B.P control in this study was still low, though, higher than those reported by some other researchers in Nigeria. Control of B.P in patients with diabetes as a co-morbid ailment was also low. Advanced age and diabetes were the two independent predictors of poorly controlled B.P in this study. Therefore, Clinicians and other health care providers should provide close attention and 
targeted B.P control on elderly diabetic hypertensive patients. Also, SBP control among these categories of patients should be prioritized, given that it was a particular problem identified in this study. Further research is needed to identify other factors negating good B.P control and suggests possible interventions to achieve better B.P control.

\section{Limitations}

This study was a cross sectional and involved patients being followed up. Therefore, the findings may not reflect the picture in the general population. Also, there might be a recall bias since the lifestyle practices of the study participants were based on self reports and performance of these behaviours was not observed. However, this study has added to the existing knowledge and provides an important entry point to understanding the burden and factors for poor B.P control in federal teaching hospital, Ido- Ekiti and other health facilities in Nigeria.

\section{Aknowledgement}

The authors would like to thank the authorities of FTH, IdoEkiti, where the study was conducted. We were also grateful to all the resident doctors who accepted to be part of the study.

\section{Authors' contribution}

AOI- Conceptualization of the study, data analysis, and drafted the manuscript

TAA- Literature review, data acquisition and analysis, review of manuscript for intellectual content

OMS- Literature review, review of manuscript for intellectual content

OEO- Data acquisition and analysis, review of manuscript for intellectual content

WOI- Data acquisition and analysis, review of manuscript for intellectual content

OOD- Data acquisition and analysis, review of manuscript for intellectual content.

\section{References}

1. Roger VL, Go AS, Donald M, Lioyd-Jones, Benjamen EJ, et al. (2012) American Heart Association Statistics committee and strokes Statistics subcommittee. Heart Disease and stroke Statistics. 2012 update: a report from American Heart Association Circulation 125: e22o.

2. Avodiwe EB, Ike SO, Nwokedieko SC (2009) Case fatality among hypertensionrelated admissions in Enugu, Nigeria. Nigerian Journal of Clinical Practice 12 153-156. Link: https://bit.ly/3ksU6G

3. Guessous I, Bochud M, Theler J, Gaspoz J, Peche A (2012) 1999-2009: Trends in prevalence, unawarenessTreatment and control of Hypertension in Geneva. PLOS one 7. Link: https://bit.ly/30xIM4K

4. Ekere AU, Yellowe BE, Umure S (2005) Mortality patterns in the accident and emergency department of an urban hospital in Nigeria Niger Jclin Pract 8:1418. Link:
5. Falase AO, Stewart S, Sliwa K (2012) Blood pressure pattern and prevalence of hypertension and hypertension related complications in Nigeria Africans: A review. World J Cardiol 4: 327-340. Link: https://bit.ly/3knmObx

6. Husain A, Al Bannay K, Bohm M (2014) Blood pressure control and predictors of uncontrolled hypertension Bahrain Medical Bulletin 36: 163-168. Link: https://bit.ly/3kiXEKZ

7. Yanq MH, Kang SY, Lee JA, Kim YS, Sung EJ, et al. (2017) The effect of lifestyle changes on blood pressure control among hypertensive patients. Korean $J$ Fam Med 38: 173-180. Link: https://bit.ly/2C75pT9

8. Asgedom SW, Gudina FK, Desee TA (2016) Assessment of blood pressure control among hypertensive patients in South West Ethiopia. PLoS One 11 e0166432. Link: https://bit.ly/30z0Ry3

9. Mutua EM, Gitonga MM, Mbuthia B. Muiruri N, cheptum JJ, et al. (2014) level of blood pressure control among hypertensive patients on follow-up in a Regional Referral Hospital in Central Kenya. Pan Afr Med J 18: 278. Link: https://bit.ly/2DKF0e1

10. Ezeala-Adikaibe BA, Mbadiwe N, Okudo G, Nwosu N, Nwobodo N, et al. (2017) Factors associated with Medication adherence among hypertensive patients in a Tertiary health center: A Cross- Sectional study. Arc Com Med Pub Health 3: 024-031. Link: https://bit.ly/3gDdmOK

11. Ojo OS, Malomo SO, Sogunle PT, Ige AM (2016) An appraisal of blood pressure control and its determinants among patients with primary hypertension seen in a primary care setting in western Nigeria. South Afri Fam Pract 1: 1-10. Link: https://bit.ly/2EXasGF

12. Igbiks T, Musa B (2012) Blood Pressure control among hypertensive patients in a Tertiary health care facility in Northern Nigeria. Res J Med Sci 6: 26-32. Link: https://bit.ly/2XC6pWQ

13. Olarewaju T, Aderibigbe A, Chijioke A, Sanya EO, Busari OA, et al. (2011) Descriptive analysis of blood pressure control among treated hypertensive patients in a tertiary hospital in Nigeria. Afr J Med Sci 40: 207-212. Link: https://bit.ly/2DMhjSD

14. Akpa MR, Alasia D, Emem- Chioma P (2008) An appraisal of hospital based blood pressure control in PortHarcourt, Nigeria. Nig Health J 8: 27-30. Link: https://bit.ly/2FOAokR

15. Araoye MO (2003) Subject selections sample size determination in Araoye $\mathrm{MO}$, Research methodology with statistics for health and social sciences Nathadex publishers 11-118.

16. Odeyinka OT, Ajayi IO (2017) Prevalence of hypertension and diabetes and their determinant among commercial drivers in Ibadan metropolis, South Western Nigeria. Nig J Cardiol 14: 75-83. Link: https://bit.ly/31uXWWu

17. Morisky DE, Green LW, Levine DM (1986) Concurrent and predictive validity of a self-reported measure of medication adherence. Med Care 24: 67-74. Link: https://bit.ly/2XEjHC2

18. Bell K, Twiggs J, Olin BR, Date IR (2015) Hypertension; the silent killer: Updated JNC-8 guideline recommendations. Alabama Pharmacy Association 344 4222. Link: https://bit.ly/3ko8oaQ

19. Saleem F, Hassali MA, Shafie AA, Atif M, Ui-haq N, et al. (2012) Disease related knowledge and quality of life: a descriptive study focusing on hypertensive population in Pakistan. South Med Rev 5: 47-52. Link: https://bit.ly/33DEUzU

20. Vanden Berg N, Meinke-Franze C, Fiss T, Hoffmann W (2013) Prevalence and determinants of controlled hypertension in a German population cohort. BMC Public Health 13: 594. Link: https://bit.ly/2XSEoKX

Copyright: @ 2020 Ibrahim AO, et al. This is an open-access article distributed under the terms of the Creative Commons Attribution License, which permits unrestricted use distribution, and reproduction in any medium, provided the original author and source are credited.

Citation: Ibrahim AO, Agbesanwa TA, Shabi OM, Omosanya OE, Ismail WO, et al. (2020) Blood pressure control and its associated factors among hypertensive patients in federal teaching hospital, ido- ekiti, Southwest Nigeria. Arch Community Med Public Health 6(2): 167-173. D0I: https://dx.doi.org/10.17352/2455-5479.000099 